\title{
The Monte Carlo Method of Mixed Radiation Field Dose Assessment in the Cytogenetic Biodosimetry
}

\author{
A. Powojska ${ }^{a}$, I. SŁoneckA ${ }^{a, b}$ And K.W. Fornalski ${ }^{c, *}$ \\ ${ }^{a}$ Warsaw University of Technology, Faculty of Physics, Koszykowa 75, 00-662 Warszawa, Poland \\ ${ }^{b}$ Central Laboratory for Radiological Protection (CLOR), Konwaliowa 7, 03-194 Warszawa, Poland \\ ${ }^{c}$ Ex-Polon Laboratory, Podleśna 81a, 05-552 Łazy, Poland
}

(Received June 2, 2018; revised version July 27, 2018; in final form August 7, 2018)

\begin{abstract}
Without any available physical measurements, absorbed doses of radiation can be assessed through the use of biological methods, of which the most common is cytogenetic biodosimetry. Evaluating the absorbed dose of mixed radiation requires determining the separate doses of each component. This paper aims to test the effectiveness of the Monte Carlo method as an alternate statistical approach for assessing absorbed doses of mixed neutron- $\gamma$ fields. It combines the iterative method with Bayesian statistics, allowing for evaluation both when the $\gamma$ to total absorbed dose ratio is known as well as when it is not. Additionally, this paper demonstrates a few of the statistical tests made possible by the Monte Carlo technique, including the distribution of damages among cells.
\end{abstract}

DOI: 10.12693/APhysPolA.134.583

PACS/topics: biodosimetry; radiation biophysics; Monte Carlo; Bayesian; dose assessment; cytogenetics

\section{Introduction}

Regardless of the cause or magnitude of a radiological incident, in its aftermath there is one aspect that has priority over others: the well-being of the affected. Estimating absorbed dose, while also keeping in mind the type of radiation and its effects on organic matter, is crucial for determining the degree of exposure as well as potentially planning treatment. This paper focuses on situations when physical dosimetry is either unavailable or insufficient, requiring the use of biodosimetric methods to assess the absorbed dose. Of these methods, cytogenetic analysis has become the most prevalent and frequently used [1].

Cytogenetic biodosimetry relies of the quantification of chromosome aberrations (such as dicentrics, rings, micronuclei, etc.) in lymphocytes taken from peripheral blood. Dose evaluations done within this study are based on dicentric chromosome assays, which are currently the preferred method for radiation biodosimetry [2]. This is mainly due to their low background level $(0.5-1 / 1000$ cells $)$ and reliability of detection.

Once the dicentric frequency is established, the absorbed dose can be calculated using coefficients from the appropriate dose-response curves. Unfortunately, this approach is insufficient when dealing with mixed radiation, which is composed of at least two types of particles. This paper focuses on mixed neutron- $\gamma$ fields, produced primarily in reactors in nuclear power plants. These institutions employ many people, working on maintaining the reactor or utilizing the energy it produces. In the case of a reactor failure, they would be at risk of being exposed to large amounts of ionizing radiation. Therefore, developing an effective method of assessing their condition is of utmost importance. Different particles have a different biological effectiveness. Both the severity and ionization mechanisms influence the number of chromosome aberrations induced by each radiation component. When scoring chromosome mutations, it is impossible to differentiate between those induced by photons and those induced by neutrons. Treating this type of mixed exposure requires determining the ratio of one component to the other. If that information is not available, it is possible to approximate it using a prior probability distribution $[3,4]$. There are several such statistical methods that can be used for assessing a mixed radiation dose, such as the iterative method or Bayesian statistics $[5,6]$.

This study aims to implement an alternative statistical method, the Monte Carlo technique, for assessing the absorbed dose of mixed neutron- $\gamma$ radiation. The iterative method has been used for this purpose on many occasions. It is an accurate method and has proven its worth many times over. Unfortunately, it cannot be employed in cases, where the exact ratio of $\gamma$ to neutron radiation is unavailable. In these cases, Bayesian statistics use probability distributions in place of exact $\gamma$-to-neutron ratios for the same calculations. The Monte Carlo method can be employed in both of these circumstances, when the ratio is known as well as when it is not. Monte Carlo is more versatile than either of the previously mentioned methods. It collects data in such a way that allows for statistical analysis of the virtually irradiated cells [6]. If needed, the distribution of neutron- and $\gamma$-induced damages among the cells can be examined [5].

\footnotetext{
* corresponding author; e-mail: krzysztof.fornalski@gmail.com
} 


\section{Methods}

\subsection{Analytical method}

The analytical method is a fully mathematical description of the commonly used so-called iterative method, recommended by the International Atomic Energy Agency (IAEA) [3], as a manual technique of assessing mixedradiation doses $[7,8]$. Its analytical form, which is much quicker and more precise, can be used when the neutron to $\gamma$ absorbed dose ratio is known

$$
\rho=\frac{D_{n}}{D_{g}}
$$

where $D_{n}$ is the neutron absorbed dose and $D_{g}$ is the $\gamma$ absorbed dose in peripheral blood lymphocytes. It is assumed, however, that the irradiation of the body is homogeneous and doses in blood are equivalent to doses in the whole body.

The $\rho$ parameter, which can vary from zero to infinity, can be replaced with $\theta$, normalized to $\langle 0,1\rangle[2,9]$ :

$$
\theta=\frac{D_{g}}{D_{g}+D_{n}}=\frac{1}{\rho+1} .
$$

Calibration curves for the quantity of chromosome aberrations (dicentrics in peripheral blood lymphocytes) from neutron and $\gamma$ irradiation are expressed as

$$
\left\{\begin{array}{l}
Y_{n}\left(D_{n}\right)=Y_{0}+\alpha D_{n}, \\
Y_{g}\left(D_{g}\right)=Y_{0}+\beta D_{g}+\gamma D_{g}^{2},
\end{array}\right.
$$

whereas the total dicentric frequency (the sum of frequencies from $\gamma$ and neutron irradiation), which is the main measured parameter, is written as

$$
Y_{\text {total }}=Y_{0}+\alpha D_{n}+\beta D_{g}+\delta D_{g}^{2} \equiv y_{f},
$$

where $Y_{0}, \alpha, \beta, \delta$ are fitting parameters of the calibration curve, which is linear-quadratic for $\gamma$ and linear for neutron irradiation [4]. All these parameters are assumed to be known and are experimentally available along with their appropriate uncertainties [5, 7], see Sect. 3. For precise parameter values, the calibration curve is obtained using physical dosimetry.

The main idea of the described analytical method is to use a system of two equations, Eq. (2) and Eq. (4), to obtain $D_{n}(\theta)$ and $D_{g}(\theta)[6,9,10]$ :

$$
\left\{\begin{array}{l}
D_{g}(\theta)=\frac{\sqrt{\left(\alpha \frac{1-\theta}{\theta}+\beta\right)^{2}+4 \gamma\left(y_{f}-Y_{0}\right)}-\left(\alpha \frac{1-\theta}{\theta}+\beta\right)}{2 \gamma}, \\
D_{n}(\theta)=\frac{1-\theta}{\theta} D_{g}(\theta) .
\end{array}\right.
$$

The proper uncertainties of doses can be calculated using e.g. exact differential method.

The benefit of the analytical approach is shortening the time required to reach a stable result in comparison to the manual iterative one $[3,8]$. Nonetheless, neither the iterative nor the analytical method can be used if the $\gamma$-to-neutron ratio is not known or known with limited likelihood.

\subsection{Enhanced analytical and Bayesian methods}

In most accidental exposures, the exact composition of the neutron- $\gamma$ field is not precisely known. In these cases, $\theta$ can be given by a prior function, $p(\theta)$ (discussed in the next subsection). The enhanced analytical method, which is thoroughly described in recently published papers $[6,10]$, builds on elements of Bayesian statistics (prior functions) as well as on the techniques of the iterative method. Thus, variables from the previous approach can be changed $\left(\theta\left(D_{x}\right)\right)$ and the probability distribution of the dose can be expressed as the function of dose, $P\left(D_{x}\right)[6,7]$. Ultimately, the dose is presented as the maximum of the $P\left(D_{x}\right)$ probability distribution [1].

However, the standard Bayesian method can also be used when the prior function, $p(\theta)$, is multiplied by the Poissonian likelihood, $L$, to get the posterior probability distribution

$$
P\left(D_{x}\right)=\int_{0}^{1} L\left(D_{x} \mid \theta\right) p(\theta) \mathrm{d} \theta,
$$

where

$$
L=\frac{\left(w y_{f}\right)^{u} \mathrm{e}^{-w y_{f}}}{u !}
$$

and $w$ is the number of cells, $u$ is the number of events (in this case chromosomal aberrations) and $y_{f}$ is the total number of aberrations, see Eq. (4) [5, 9].

\subsection{Prior functions}

A prior distribution is a probability density function (PDF) used to estimate the most likely value of the observed parameter, in this case $\theta$ [6]. Selecting a prior depends on the current degree of uncertainty about the radiation incident in question [11].

The first approach is finding a simple and intuitive prior, where there is just a little information about the parameter $[11,12]$. The special case of the Beta distribution is an example of that prior, where a general assumption is made that the absorbed dose was half neutrons, half $\gamma$ photons

$$
p(\theta)=\theta-\theta^{2} \text {. }
$$

The second approach pertains to fully informative priors, selected based on previous knowledge, experience and/or data. It is a more subjective approach but incorporating such information into data analysis can provide a more precise result [11]. An example of that informative prior is the Gaussian distribution for $\rho$ however in practical applications, the scaled Gaussian distribution in $\theta$ coordinates (see Eq. (2)) is preferred [13]:

$$
p(\theta)=\frac{1}{\sqrt{2 \pi} \sigma_{\rho} \theta^{2}} \exp \left[\frac{-1}{2 \sigma_{\rho}^{2}}\left(\left(\frac{1}{\theta}-1\right)-\hat{\rho}\right)^{2}\right],
$$

where $\hat{\rho} \pm \sigma_{\rho}$ and $\theta$ parameters were presented in Eq. (2).

\subsection{Monte Carlo}

The Monte Carlo (MC) methods are a class of computing algorithms that rely on repeated random sampling as a way of statistically evaluating mathematical functions. The particular variation of the MC technique used in this study is a combination of the classical iterative 


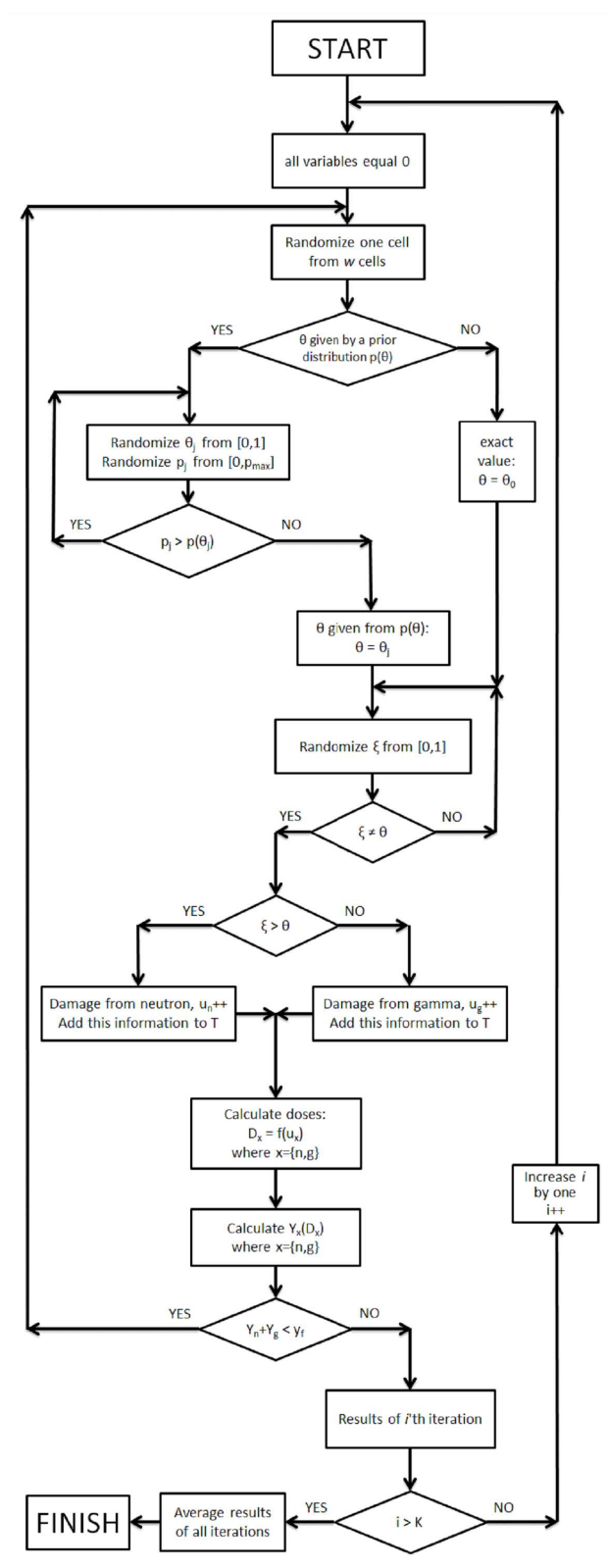

Fig. 1. The scheme of the Monte Carlo algorithm [6].

and the enhanced analytical methods. It requires a dedicated computer program implementing the algorithm outlined in Fig. 1 [6], which was created as part of this study ${ }^{\dagger}$.

This program has two major loops: the outer loop, repeated over $K$ iterations (each iteration is a single complete simulation) and the inner loop that repeats over $w$ cells and contains the core of the algorithm. At the start of the program, the user must provide some initial information (taken from experiments or measurements): the measured dicentric count $\left(y_{f}=Y_{\text {total }}\right)$ and parameters for the calibration curves (Eq. (3)).

\footnotetext{
$\dagger_{\text {www.clor.waw.pl/publikacje.html }}$
}

The user can then choose to either provide an exact $\theta$ or use a probability distribution to approximate its value. This program offers 8 priors to choose from with modifiable parameters. Within a single iteration of the inner loop, a cell is randomized from $w$ cells. If the option of using a prior, $p(\theta)$, was chosen, then a Monte Carlo randomization helps to estimate $\theta$ using random sampling. If an exact $\theta$ was given by the user, the above steps are omitted.

In order to determine whether a cell was altered by a neutron or by a $\gamma$ photon, an additional variable, $\xi$, is randomized from $[0,1]$ and then compared to $\theta(\xi \neq \theta)$. If it is higher than $\theta$, then the damage is classified as neutron induced (the $u_{n}$ variable is increased by one), otherwise the damage came from $\gamma$ radiation (the $u_{g}$ variable is increased by one). The absorbed dose is then calculated as [6]:

$$
D_{x}=f\left(u_{x}\right) \approx \operatorname{const}_{x} \cdot u_{x},
$$

where $u_{x}$ is the amount of damages induced by a specific radiation component, $x=\{g, n\}$ while const $_{g}$ and const $_{n}$ are calculated during the calibration of the program. Equations (3) are then used to determine the aberration frequency. The inner loop repeats until the total aberration frequency $\left(Y_{g}+Y_{n}\right)$ is no longer smaller than $y_{f}$. The doses and aberration frequencies are calculated $K$-times and averaged.

Two main advantages of the MC technique over other methods stem from its stochastic approach in estimating dose [6]:

- Statistical analysis - the construction of this method allows for various types of statistical tests, for example a type of "damage time line" can be created or different distribution of cell parameters can be obtained. It is also possible to study correlations between different variables;

- Probabilistic results - the algorithm provides results as well as how likely they are.

Before the above algorithm can be of practical use, it must be calibrated, which in this case is fairly simple. The idea is to obtain appropriate values of const $_{g}$ and const $_{n}$ from Eq. (9). This was done by modifying the constants and running the calculations until the results generated by the program match the experimental data. This will be further discussed in Sect. 3.1.

\section{Results \\ 3.1. Calibration}

Equation (9) is an empirical relationship, based more on observation than pure theory, and is therefore assumed to be linear. Thus, the constants const $_{g}$ and const $_{n}$ must be assigned a unit. Based on the knowledge that $u_{x}$ represents the number of dicentrics within the virtual cell group $(w=1000)$, as well as the fact that $D_{x}$ is expressed in grays [Gy], the right and left side of the equation can be balanced by assigning const $_{g}$ and const $_{n}$ the unit $[\mathrm{Gy} / \mathrm{dic}]$. 
TABLE I

Results collected after the irradiation of samples with mixed $\mathrm{n}+\gamma$ radiation $(\theta=0.92 \pm 0.02)$

\begin{tabular}{c|c|c|c|c|c|c}
\hline \hline \multirow{2}{*}{$\begin{array}{c}\text { Sample } \\
\text { number }\end{array}$} & \multicolumn{2}{|c|}{ Total } & \multicolumn{2}{c|}{ Gamma } & \multicolumn{2}{c}{ Neutrons } \\
\cline { 2 - 7 } & $\begin{array}{c}D_{t} \\
{[\mathrm{~Gy}]}\end{array}$ & $\begin{array}{c}Y_{t} \\
{[\text { dic/cell] }}\end{array}$ & $\begin{array}{c}D_{g} \\
{[\mathrm{~Gy}]}\end{array}$ & $\begin{array}{c}Y_{g} \\
{[\text { dic/cell] }}\end{array}$ & $\begin{array}{c}D_{n} \\
{[\mathrm{~Gy}]}\end{array}$ & $\begin{array}{c}Y_{n} \\
{[\text { dic/cell] }}\end{array}$ \\
\hline 1 & 0 & 0.001 & 0 & 0.0005 & 0 & 0.0005 \\
2 & 0.25 & 0.014 & 0.23 & 0.006 & 0.02 & 0.075 \\
3 & 0.5 & 0.032 & 0.46 & 0.018 & 0.04 & 0.0145 \\
4 & 0.75 & 0.056 & 0.69 & 0.035 & 0.06 & 0.021 \\
5 & 0.9 & 0.076 & 0.828 & 0.049 & 0.072 & 0.027 \\
6 & 1 & 0.086 & 0.92 & 0.059 & 0.08 & 0.027 \\
7 & 1.5 & 0.165 & 1.38 & 0.123 & 0.12 & 0.042
\end{tabular}

Calibration of the constants was done based on blood samples irradiated in channel $\mathrm{H} 8$ of the Maria Reactor, located in the National Centre for Nuclear Research (NCBJ), Swierk-Otwock, Poland. All parameters of the radiation beam are described in detail in the paper by Golnik et al. [14]. Additionally, reference data (Table I) $[5,7]$ was provided by the Central Laboratory for Radiological Protection (CLOR), Warsaw, Poland, which analysed and assessed the blood samples through meth- ods of biological dosimetry. The dose-response calibration curves used for determining the constants (Eqs. (10) and (11)) were fitted based on the results of the analysis

$$
\begin{aligned}
& Y_{g}=(0.0005 \pm 0.0001)+(0.0119 \pm 0.0027) D_{g} \\
& \quad+(0.0557 \pm 0.0016) D_{g}^{2} \\
& Y_{n}=(0.0005 \pm 0.0001)+(0.354 \pm 0.003) D_{n} .
\end{aligned}
$$

Channel H8 of the Maria Reactor generates a specific type of neutron and $\gamma(n+\gamma)$ radiation, where the $\gamma$ component of the absorbed dose makes up $92 \%$ percent of the total, therefore, the radiation to which the samples were exposed had a $\theta$ equal to 0.92 [7].

At first, it was assumed that the two constants share the same value (const $_{g, n} \approx 0.012$, taken from [6]). During calibration it was found that the program generates more precise results (Table II) when provided with two different values of that parameter. Although the difference is minimal, it is significant enough to influence the accuracy of the algorithm. Two separate constants suggest possible non-linearity of the $D_{x}$ formula (Eq. (9)), but several tests showed that linear $D_{g}$ and $D_{n}$ with different const $_{g}$ and const $_{n}$ produce the best results. The results of the calibration are presented in Table II.

TABLE II

\begin{tabular}{c|c|c|c|c|c|c|c}
\hline \hline & \multicolumn{7}{c}{ Sample number } \\
\cline { 2 - 7 } & 1 & 2 & 3 & 4 & 5 & 6 & 7 \\
\hline const $_{g}[\mathrm{~Gy} / \mathrm{dic}]$ & 0.01201 & 0.01201 & 0.01270 & 0.01255 & 0.01190 & 0.01255 & 0.01201 \\
$\operatorname{const}_{n}[\mathrm{~Gy} / \mathrm{dic}]$ & 0.01200 & 0.01200 & 0.01220 & 0.01200 & 0.01230 & 0.01200 & 0.01200 \\
\hline \multicolumn{7}{|c|}{ Average } \\
\hline
\end{tabular}

\subsection{Program results}

Figure 2 presents the user interface of the program containing the Monte Carlo algorithm.

The algorithm was verified using experimental data collected in conditions where the characteristics of the neutron- $\gamma$ beam were known. The samples used are presented in Table III.

Each blood sample was irradiated in the Maria Reactor in Swierk-Otwock with a mixed $n+\gamma$ radiation $(\theta=0.92)$. Samples 1 and 3 were collected during the same experiment as the data that was used for calibration (Sect. 3.1), while sample 2 was acquired from [9].

Using the Monte Carlo program, the doses and aberration frequencies were assessed in several ways (using the standard calibration curves, Eqs. (10) and (11)):

1. with an exact $\theta=0.92$ and $\sigma_{\theta}=0.02$,

2. with a Gaussian prior: $\theta=0.92, \sigma_{\theta}=0.02$,

3. with a scaled Gaussian prior: $\rho=0.086, \sigma_{\rho}=0.02$.

The results were further validated by comparing them to results obtained using three other statistical methods: iterative, analytical, and Bayesian with a Gaussian prior.

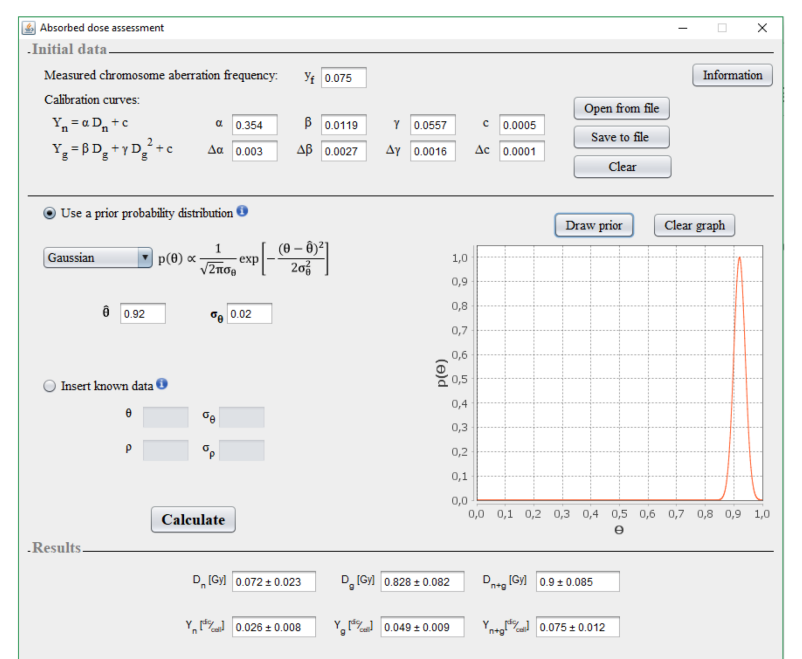

Fig. 2. Graphical User Interface (GUI) of the program.

For each method $\theta=0.92$ and $\sigma_{\theta}=0.02$. These calculations were done by a separate program ${ }^{\dagger}$ that was created as part of a diploma thesis [15] written by Łukasik. The results are presented in Tables IV-IX. 
Experimental data used for program

TABLE III verification

\begin{tabular}{c|c|c|c|c|c|c}
\hline \hline $\begin{array}{c}\text { Sample } \\
\text { number }\end{array}$ & $\begin{array}{c}D_{t} \\
{[\mathrm{~Gy}]}\end{array}$ & $\begin{array}{c}D_{g} \\
{[\mathrm{~Gy}]}\end{array}$ & $\begin{array}{c}D_{n} \\
{[\mathrm{~Gy}]}\end{array}$ & $\begin{array}{c}Y_{t} \\
{[\mathrm{dic} / \text { cell }]}\end{array}$ & $\begin{array}{c}Y_{g} \\
{[\mathrm{dic} / \text { cell] }}\end{array}$ & $\begin{array}{c}Y_{n} \\
{[\mathrm{dic} / \text { cell }]}\end{array}$ \\
\hline 1 & 0.1 & 0.092 & 0.008 & 0.005 & 0.002 & 0.003 \\
\hline 2 & 0.85 & 0.782 & 0.068 & 0.07 & - & - \\
\hline 3 & 2 & 1.84 & 0.16 & 0.27 & 0.211 & 0.059
\end{tabular}

Sample 1 - doses

TABLE IV

\begin{tabular}{l|c|c|c}
\hline \hline Method & $D_{t}[\mathrm{~Gy}]$ & $D_{g}[\mathrm{~Gy}]$ & $D_{n}[\mathrm{~Gy}]$ \\
\hline \multicolumn{4}{|c}{ Monte Carlo } \\
no prior & $0.101 \pm 0.061$ & $0.093 \pm 0.061$ & $0.008 \pm 0.006$ \\
Gaussian & $0.099 \pm 0.060$ & $0.091 \pm 0.060$ & $0.008 \pm 0.006$ \\
scaled Gaussian & $0.101 \pm 0.060$ & $0.093 \pm 0.060$ & $0.008 \pm 0.006$ \\
\hline iterative & - & $0.084 \pm 0.043$ & $0.007 \pm 0.004$ \\
analytical & - & $0.094 \pm 0.052$ & $0.008 \pm 0.004$ \\
Bayesian & - & $0.093 \pm 0.044$ & $0.008 \pm 0.004$
\end{tabular}

Sample 1 - aberration frequencies

TABLE V

\begin{tabular}{l|c|c|c}
\hline \hline Method & $Y_{t}$ [dic/cell] & $Y_{g}[\mathrm{dic} /$ cell $]$ & $Y_{n}[\mathrm{dic} /$ cell] \\
\hline \multicolumn{4}{|c}{ Monte Carlo } \\
no prior & $0.005 \pm 0.003$ & $0.002 \pm 0.001$ & $0.003 \pm 0.002$ \\
Gaussian & $0.005 \pm 0.003$ & $0.002 \pm 0.001$ & $0.003 \pm 0.002$ \\
scaled Gaussian & $0.005 \pm 0.003$ & $0.002 \pm 0.001$ & $0.003 \pm 0.002$ \\
\hline iterative & - & $0.002 \pm 0.001$ & $0.003 \pm 0.002$ \\
analytical & - & $0.002 \pm 0.001$ & $0.003 \pm 0.002$ \\
Bayesian & - & $0.002 \pm 0.001$ & $0.003 \pm 0.002$
\end{tabular}

Sample 2 - doses

TABLE VI

\begin{tabular}{l|c|c|c}
\hline \hline Method & $D_{t}[\mathrm{~Gy}]$ & $D_{g}[\mathrm{~Gy}]$ & $D_{n}[\mathrm{~Gy}]$ \\
\hline \multicolumn{4}{|c}{ Monte Carlo } \\
no prior & $0.862 \pm 0.089$ & $0.795 \pm 0.086$ & $0.068 \pm 0.024$ \\
Gaussian & $0.861 \pm 0.085$ & $0.792 \pm 0.082$ & $0.069 \pm 0.023$ \\
scaled Gaussian & $0.863 \pm 0.084$ & $0.796 \pm 0.081$ & $0.067 \pm 0.023$ \\
\hline iterative & - & $0.796 \pm 0.199$ & $0.069 \pm 0.027$ \\
analytical & - & $0.798 \pm 0.097$ & $0.069 \pm 0.021$ \\
Bayesian & - & $0.798 \pm 0.207$ & $0.068 \pm 0.023$
\end{tabular}

Sample 2 - aberration frequencies

TABLE VII

\begin{tabular}{l|c|c|c}
\hline \hline Method & $Y_{t}$ [dic/cell] & $Y_{g}[$ dic/cell] & $Y_{n}$ [dic/cell] \\
\hline \multicolumn{4}{|c}{ Monte Carlo } \\
no prior & $0.07 \pm 0.012$ & $0.046 \pm 0.009$ & $0.024 \pm 0.008$ \\
Gaussian & $0.07 \pm 0.012$ & $0.045 \pm 0.009$ & $0.025 \pm 0.008$ \\
scaled Gaussian & $0.07 \pm 0.012$ & $0.046 \pm 0.008$ & $0.024 \pm 0.008$ \\
\hline iterative & - & $0.045 \pm 0.022$ & $0.025 \pm 0.010$ \\
analytical & - & $0.045 \pm 0.010$ & $0.025 \pm 0.008$ \\
Bayesian & - & $0.045 \pm 0.021$ & $0.024 \pm 0.008$
\end{tabular}

The program also provides results in the form of graphs. Some examples are shown in Figs. 3 and 4. The first shows the results obtained for sample 2
Sample 3 - doses

TABLE VIII

\begin{tabular}{l|c|c|c}
\hline \hline Method & $D_{t}[\mathrm{~Gy}]$ & $D_{g}[\mathrm{~Gy}]$ & $D_{n}[\mathrm{~Gy}]$ \\
\hline \multicolumn{4}{|c}{ Monte Carlo } \\
no prior & $2.011 \pm 0.077$ & $1.853 \pm 0.066$ & $0.158 \pm 0.040$ \\
Gaussian & $2.011 \pm 0.076$ & $1.852 \pm 0.065$ & $0.158 \pm 0.039$ \\
scaled Gaussian & $2.012 \pm 0.076$ & $1.856 \pm 0.065$ & $0.156 \pm 0.039$ \\
\hline iterative & - & $1.847 \pm 0.247$ & $0.161 \pm 0.057$ \\
analytical & - & $1.850 \pm 0.125$ & $0.161 \pm 0.054$ \\
Bayesian & - & $1.850 \pm 0.218$ & $0.161 \pm 0.041$
\end{tabular}

Sample 3 - aberration frequencies

TABLE IX

\begin{tabular}{l|c|c|c}
\hline \hline Method & $Y_{t}$ [dic/cell] & $Y_{g}$ [dic/cell] & $Y_{n}[$ dic/cell] \\
\hline \multicolumn{4}{|c}{ Monte Carlo } \\
no prior & $0.271 \pm 0.022$ & $0.214 \pm 0.016$ & $0.057 \pm 0.014$ \\
Gaussian & $0.271 \pm 0.021$ & $0.214 \pm 0.016$ & $0.057 \pm 0.014$ \\
scaled Gaussian & $0.271 \pm 0.021$ & $0.215 \pm 0.016$ & $0.056 \pm 0.014$ \\
\hline iterative & - & $0.213 \pm 0.055$ & $0.057 \pm 0.023$ \\
analytical & - & $0.213 \pm 0.029$ & $0.057 \pm 0.019$ \\
Bayesian & - & $0.213 \pm 0.049$ & $0.059 \pm 0.015$
\end{tabular}

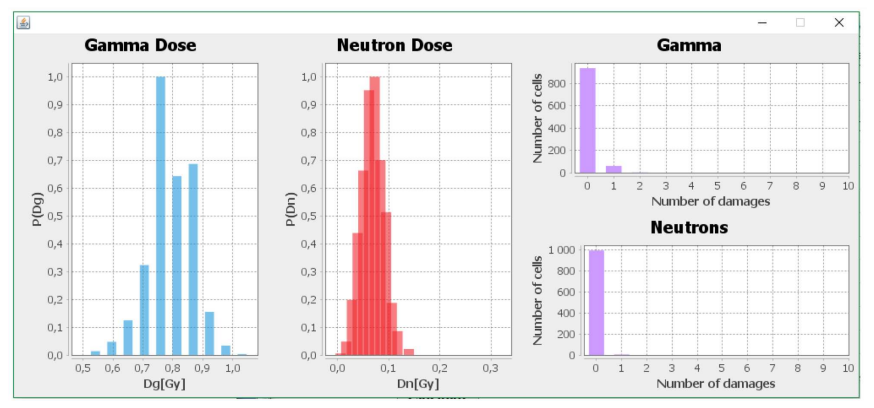

Fig. 3. Results for sample 2: $y_{f}=0.07, \theta=0.92$, $\sigma_{\theta}=0.02$.

using the Gaussian prior $\left(\theta=0.92, \sigma_{\theta}=0.02\right)$, whereas the latter contains results for a higher measured aberration frequency $\left(y_{f}=20\right)$ calculated using the Beta prior.

Monte Carlo statistics rely on random numbers. Because of this, each iteration produces a slightly different absorbed dose. The plots located on the left in Figs. 3 and 4 are frequency graphs representing how many times a dose appeared during all of the iterations. The data has been normalized, so the neutron and $\gamma$ doses could be shown on the same scale. The expected shape of these graphs is the Gaussian distribution but because of the random nature of this method as well as the data collection mechanism, the actual shapes are not exact. For the purpose of this program, it is assumed that the doses create a symmetrical Gaussian distribution. The histograms on the right represent the number of cells containing a certain amount of dicentrics. The distribution of damages can be modelled as a Poisson distribution, which becomes more visible with higher doses, as illustrated in Fig. 4. 


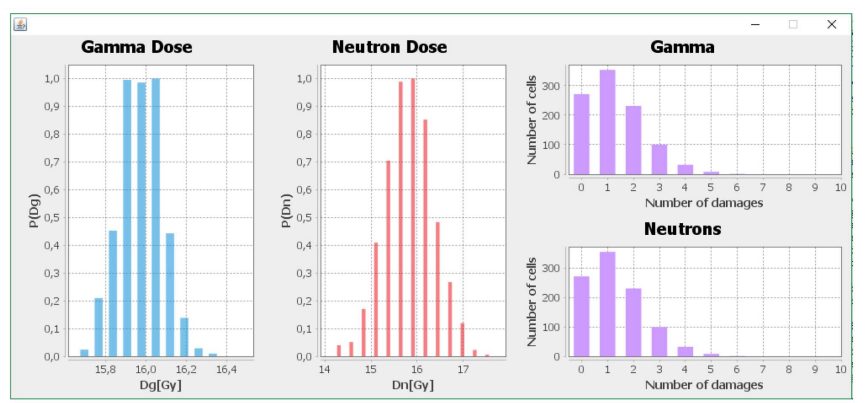

Fig. 4. Results for $y_{f}=20$ using the Beta prior $(a=2$, $b=2)$.

\subsection{Results' analysis}

The statistical parameter $E_{n}$ was used to assess the integrity of the results.

$$
E_{n}=\frac{x-X}{\sqrt{U_{\text {lab }}^{2}+U_{\text {ref }}^{2}}},
$$

where $x$ - the result calculated using the MC method, $X$ - the proper value (obtained using the reference method - in this case the iterative method), $U_{\text {lab }}-\mathrm{MC}$ result uncertainty, $U_{\text {ref }}$ - proper value uncertainty.

The comparison results are satisfactory when $\left|E_{n}\right| \leq 1$. The results are presented in Tables X and XI.

$E_{n}$ values for $\gamma$ dose $D_{g}$

TABLE X

\begin{tabular}{c|l|c}
\hline \hline Sample number & Method & $\left|E_{n}\right|$ \\
\hline \multirow{3}{*}{1} & no prior & 0.121 \\
& Gaussian prior & 0.095 \\
& scaled Gaussian & 0.122 \\
\hline \multirow{3}{*}{2} & no prior & 0.005 \\
& Gaussian prior & 0.019 \\
& scaled Gaussian & 0.000 \\
\hline \multirow{3}{*}{3} & no prior & 0.023 \\
& Gaussian prior & 0.020 \\
& scaled Gaussian & 0.035
\end{tabular}

$E_{n}$ values for neutron dose $D_{n}$

TABLE XI

\begin{tabular}{c|l|c}
\hline \hline Sample number & Method & $\left|E_{n}\right|$ \\
\hline \multirow{3}{*}{1} & no prior & 0.139 \\
& Gaussian prior & 0.139 \\
& scaled Gaussian & 0.139 \\
\hline \multirow{3}{*}{2} & no prior & 0.028 \\
& Gaussian prior & 0.000 \\
& scaled Gaussian & 0.056 \\
\hline \multirow{3}{*}{3} & no prior & 0.043 \\
& Gaussian prior & 0.043 \\
& scaled Gaussian & 0.072
\end{tabular}

\section{Discussion}

Biological dosimetry methods described in the presented work allow assessing the dose absorbed by the human body based on biological markers created as a result of ionizing radiation interacting with the body. The most accurate test to assess the dose is the analysis of dicentric chromosomes (dicentrics), formed in peripheral blood lymphocytes, by measuring their frequency, which is proportional to the absorbed dose (physically measured: linear-quadratic dependence in the case of low LET-radiation and linear dependence for high LETradiation). Due to the similarities in the levels of change in chromosome structures (dicentrics) after in vivo and in vitro irradiation, it is possible to assess the absorbed dose by comparing the effects occurring after real exposure with previously determined in vitro dose-effect curves. The curves describe the dependence of the dicentric frequency found in analysed blood samples on the absorbed dose (for a specific source of radiation). Doseeffect curves are obtained by irradiating peripheral blood samples from several donors with different doses (which takes place in strictly controlled experimental conditions, such as dose rate, measured by physical equipment [14]) and then examining the frequency of dicentrics in isolated blood lymphocytes.

The results of dicentric analysis are used in the classical iterative method, which is recommended by the International Atomic Energy Agency as a simple and trustworthy method for assessing mixed-radiation doses. It was also implemented in CLOR and accredited as a standard method in radiation biodosimetry. It is quite accurate but often requires many iterations and therefore calculations can be lengthy. This method can be modified into the analytical solution proposed recently by us [10], which removes the need for a large number of repetitions and is nothing more than an analytical description of the iterative method, thus, it gives the same results (with better precision). The primary set-back of both of these methods is that neither of them can be used if the $\gamma$ to-neutron ratio is not precisely known. The enhanced analytical method fills this gap [10]. Building on some elements of Bayesian statistics, it allows for the unknown ratio to be presented as a prior function.

The presented Monte Carlo method combines the classical iterative and enhanced analytical approaches, and this is the main reason why both methods were mentioned within this paper. The created algorithm is straightforward in both understanding and implementation. It allows for dose assessment when the $\gamma$-to-neutron ratio is known, as well as when it must be estimated via a prior function. An important benefit of using the Monte Carlo method is that the manner in which data is collected leaves room for statistical testing of all the parameters, which is impossible in previous methods. More than that the presented algorithm can be easily implemented into a wider model of radiation risk estimation [16]. This approach is therefore different from typical deterministic models. Since it is based around random sampling, the results of each iteration are slightly different and therefore the final results also vary between runs, which is typical for stochastic approaches. These differences are minimal and represent the margin of statistical error, 
which is rather important information. Partly due to the nature of this Monte Carlo algorithm, the frequency graphs illustrating the distribution of doses throughout the iterations are not always symmetrical. As discussed in Sect. 2.4, each iteration of the inner loop continues until the sum of $Y_{g}$ and $Y_{n}$ is no longer smaller than the measured $y_{f}$. For low values of aberration frequencies, there are not only fewer iterations of this loop, but also the calculated doses are not as varied as with higher aberration frequencies. Therefore, the graphs that are meant to show the frequency distribution of doses do not have enough values to produce a proper histogram. The threshold value below which this occurs varies depending on the calibration curves and the value of $\theta$.

The results acquired during this study were compared to a reference method (iterative one) using the $E_{n}$ parameter in order to assess their validity. All of the comparison results were well below 1 , therefore the necessary requirement was satisfied, which means that the proposed Monte Carlo method is accurate and can be successfully used.

\section{Acknowledgments}

This paper is part of an engineer thesis written by the first author — the student of Faculty of Physics at Warsaw University of Technology in the academic year 2016/2017, as a part of scientific program $\mathrm{SP} / \mathrm{J} / 16 / 143339 / 8$, "Technologies Supporting Development of Safe Nuclear Power Engineering" (NCBiR).

Authors wish to thank Dr. Maria Kowalska (CLOR) for substantial help, all remarks and valuable discussions.

\section{References}

[1] A. Giovanetti, A. Scura, G. Aversa Biological Dosimetry: How to Measure the Absorbed Dose in Different Scenarios, ENEA, 2012.

[2] R.C. Wilkins, H. Romm, U. Oestreicher, L. Marro, M.A. Yoshida, Y. Suto, P.G. Prasanna, Radiat. Measur. 46, 923 (2011).
[3] International Atomic Energy Agency, Cytogenetic Dosimetry: Applications in Preparedness for and Response to Radiation Emergencies, technical report, International Atomic Energy Agency, 2011.

[4] Biological Dosimetry IAEA, "Chromosomal aberration analysis for dose assessment", Tech. Rep. Series (260), 1986.

[5] I. Pacyniak, K.W. Fornalski, M. Kowalska, in: Proc. Second Int. Conf. on Radiation and Dosimetry in Various Fields of Research, RAD 2014, Ed.: G. Ristić, University of Niš (Serbia), Niš 2014, p. 49.

[6] K.W. Fornalski, arXiv:1412.2048, 2014.

[7] I. Słonecka, M.Sc. Thesis, Warsaw University of Technology, 2014, Accessed: 2016-05-20.

[8] M. Szłuińska, A.A. Edwards, D.C. Lloyd, Statistical Methods for Biological Dosimetry, Health Protection Agency, Radiation Protection Division, 2005.

[9] I. Pacyniak, K.W. Fornalski, M. Kowalska, Postępy Fizyki 66, 13 (2015).

[10] I. Słonecka, K. Łukasik, K.W. Fornalski, Radiat. Environm. Biophys. 57, 195 (2018).

[11] M.E. Glickman, D.A. van Dyk, Meth. Mol. Biol. 404 , 319 (2007).

[12] M.A.T. Figueiredo, "Lecture notes on Bayesian estimation and classification", Instituto de Telecomunicacoes-Instituto Superior Tecnico, Lisboa 2004.

[13] R.S. Brame, P.G. Groer, Radiat. Protect. Dosim. 104, 61 (2003).

[14] N. Golnik, M. Gryziński, M. Kowalska, K. Meronka, P. Tulik, Radiat. Protect. Dosim. 161, 196 (2014).

[15] K. Łukasik, Bachelor's Thesis, Warsaw University of Technology, 2016.

[16] L. Dobrzyński, K.W. Fornalski, Y. Socol, J.M. Reszczyńska, Radiat. Res. 186, 396 (2016). 